\title{
Infecção natural de Triatoma vitticeps (Stal, 1859) por flagelados morfologicamente semelhantes a Trypanosoma cruzi (Chagas, 1909) no Estado do Espírito Santo
}

\author{
Natural infection of Triatoma vitticeps (Stal, 1859) with flagellates morphologically \\ similar to Trypanosoma cruzi (Chagas, 1909) \\ in Espírito Santo State
}

\author{
Claudiney Biral dos Santos ${ }^{1,2}$, Gustavo Rocha Leite ${ }^{1,3}$, Gabriel Eduardo Melim Ferreira ${ }^{1,4}$ \\ e Adelson Luiz Ferreira ${ }^{1,5}$
}

\begin{abstract}
RESUMO
Espécimes adultos de Triatoma vitticeps são capturados freqüentemente por moradores em áreas rurais do Estado do Espírito Santo, Brasil. Com o objetivo de determinar o índice de infecção natural desta espécie, examinamos os dejetos de 116 espécimes silvestres, capturados em 27 municípios do estado, após repasto sanguíneo em ave e dejeção espontânea. Destes, 100 (86,2\%) estavam infectados por flagelados morfologicamente semelhantes a Trypanosoma cruzi. Detectamos índices de infecção natural de Tritoma vitticeps superiores ao de estudos anteriores. A baixa incidência da doença de Chagas no estado se deve provavelmente a dejeção tardia deste vetor visto que trabalhos sobre especificidade alimentar demonstraram presença marcante de Tritoma vitticeps no intradomicilio e contato freqüente com o homem. O elevado índice de infecção natural observado reforça a necessidade de se manter a vigilância entomológica sobre este triatomíneo.
\end{abstract}

Palavras-chaves: Triatoma vitticeps. Infecção natural. Trypanosoma cruzi. Espírito Santo. Brasil.

\begin{abstract}
Adult specimens of Triatoma vitticeps are frequently captured by residents in rural areas of the State of Espírito Santo, Brazil. With aim of determining the natural infection rate of this species, we examined the excrement of 116 wild specimens, captured in 27 municipal districts of the state, after blood meal in chicken and spontaneous excretion. Of these, 100 (86,2\%) were infected with flagellates morphologically similar to Trypanosoma cruzi. Our results showed natural infection rates of Tritoma vitticeps superior to previous studies. The low incidence of Chagas disease in the state is probably due to late excretion in this vector, given that works of alimentary specificity demonstrated the presence of Tritoma vitticeps in intradomiciliary and frequent contact with humans. The high rate of natural infection observed reinforces the need for sustained entomologic surveillance on this triatomine.
\end{abstract} Key-words: Triatoma vitticeps. Natural infection. Trypanosoma cruzi. Espírito Santo. Brazil.

Espécimes adultos de Triatoma vitticeps (Stal, 1859) procedentes de florestas remanescentes são capturados freqüentemente por moradores no ambiente domiciliar de áreas rurais no Estado do Espírito Santo, Brasil ${ }^{10}$. Inquéritos sorológicos têm demonstrado prevalência baixa de doença de Chagas no estado, sendo os poucos casos autóctones de transmissão humana de Trypanosoma cruzi (Chagas, 1909) atribuídos a $T$. vitticeps ${ }^{12}{ }^{12}$. A espécie também é incriminada como transmissor deste parasita no Estado do Rio de Janeiro ${ }^{7}$.

Estudos realizados anteriormente no Espírito Santo, por Santos e cols ${ }^{10}$, Barros e cols ${ }^{1}$, Sessa e coll ${ }^{11}$ e Dias e cols ${ }^{4}$, demonstraram resultados discrepantes de 44\%, 5,3\%, 64,7\% e 60,8\%, respectivamente, dos índices de infecção natural de T. vitticeps por flagelados morfologicamente semelhantes a T. cruzi.

\footnotetext{
1. Medicina Tropical da Universidade Federal do Espírito Santo, Vitória, ES. 2. Fundação Nacional de Saúde, Vitória, ES. 3. Biologia Animal da Universidade Federal do Espírito Santo, Vitória, ES. 4. Biologia Parasitária do Instituto Oswaldo Cruz, Rio de Janeiro, RJ. 5. Escola Superior de Ciências da Santa Casa de Misericórdia de Vitória, Vitória, ES.

Endereço para correspondência: Dr. Claudiney Biral dos Santos. Dept ${ }^{0}$ de Patologia/CB/UFES. Av. Marechal Campos 1468, $29040-090$ Vitória, ES, Brasil.

Telefax: 5527 3324-2038

e-mail: claudiney@ppgcf.ufes.br

Recebido em 3/8/2004

Aceito em 30/8/2005
} 
Neste trabalho, avaliamos o índice de infecção natural de espécimes silvestres de $T$. vitticeps capturados em municípios diversos do Espírito Santo.

No período de dezembro de 2001 a dezembro de 2003, 598 espécimes adultos de $T$. vitticeps foram remetidos pelas Unidades Sanitárias Regionais do Estado do Espírito Santo para o laboratório de Entomologia da Secretaria de Estado da Saúde que se localiza no município de Vitória. Os espécimes foram coletados por moradores nas suas residências em áreas rurais do estado.

Identificamos os espécimes remetidos conforme chave de Lent e $\mathrm{col}^{6}$, os acondicionamos em gaiolas individuais e os observamos durante dois dias, de modo que selecionássemos para o experimento somente os que estavam aparentemente sadios e aptos para a alimentação, de forma que seus dejetos fossem coletados espontaneamente após o repasto.

Dos 598 espécimes remetidos, selecionamos 116, tendo estes sido capturados em 81 localidades diferentes, distribuídas por 27 municípios do estado (Figura 1). Alimentamos os triatomíneos selecionados individualmente em ave (Gallus gallus domesticus) e após a dejeção espontânea realizamos pesquisa a fresco de flagelados em seus dejetos.

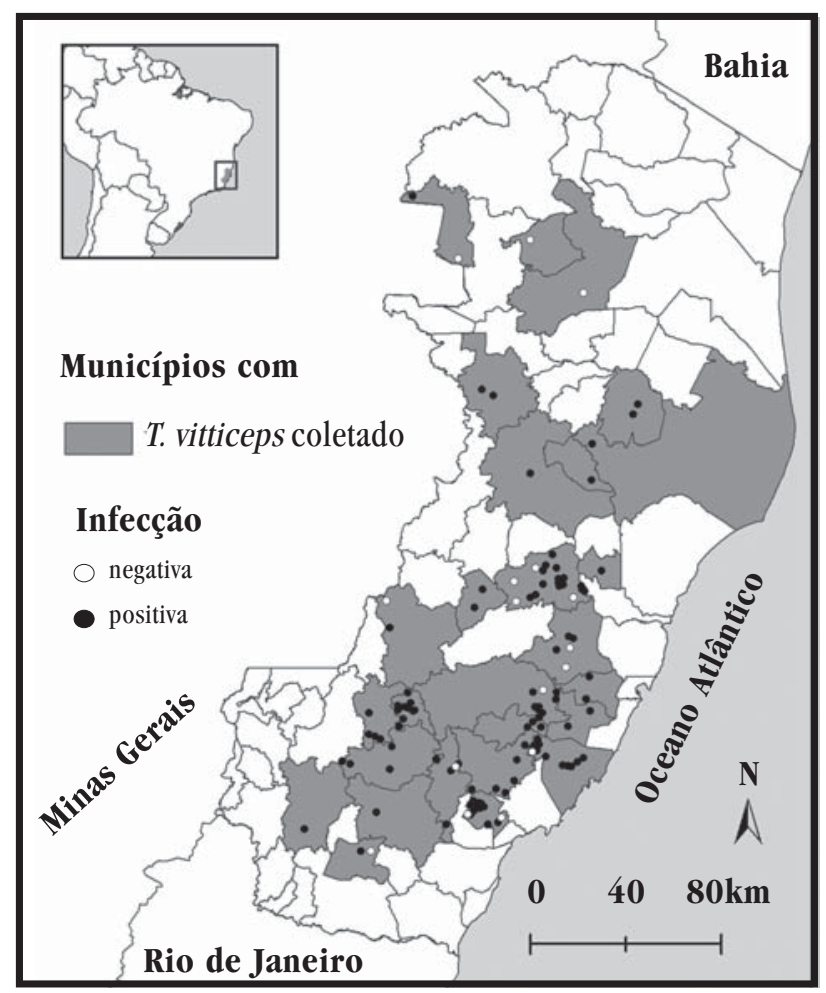

Figura 1 - Mapa do Estado do Espírito Santo, com os municípios positivos para a presença de Triatoma vitticeps e localidades onde os 116 espécimes foram coletados indicando se infectados por flagelados semelhantes a Trypanosoma cruzi entre o período de 2001 a 2003.

Dos 116 espécimes examinados (35 machos e 81 fêmeas), $100(86,2 \%)$ estavam infectados naturalmente por flagelados semelhantes a $T$. cruzi, sendo destes $30(85,7 \%)$ machos e 70 (86,4\%) fêmeas. Ainda, 55 (47,4\%) foram capturados em dormitórios de residências, sendo que destes, 47 (85\%) estavam infectados. Os 61 espécimes restantes foram capturados na sala $(14,7 \%)$, cozinha $(9,5 \%)$ e outros cômodos $(28,4 \%)$.

Mesmo com a distribuição dos municípios amostrados neste estudo sendo semelhante ao de estudos realizados anteriormente por Sessa $\operatorname{col}^{11}$ e Dias $\operatorname{cols}^{4}\left(\mathrm{x}^{2}=0,011 ; \mathrm{gl}=1\right.$; $\mathrm{p}=0,594)$, nossos resultados mostraram índices de infecção natural de $T$. vitticeps superiores (Tabela 1). As diferenças nos índices podem ter ocorrido em função de em nosso estudo termos realizado o exame a partir da dejeção espontânea, após o repasto sanguíneo. Tal método se mostrou eficiente na detecção dos flagelados, sendo que Sherlock ${ }^{13}$ sugere que o conteúdo alimentar no estômago do inseto pressiona os túbulos de Malpighi liberando excretas ricos em tripomastigotas.

Tabela 1 - Resultados dos índices de infecção natural de Triatoma vitticeps encontrados por diferentes autores no Estado do Espírito Santo.

\begin{tabular}{lcccc}
\hline $\begin{array}{l}\text { Exemplares } \\
\text { examinados }\end{array}$ & $\begin{array}{c}\text { Índice de } \\
\text { infecção }(\%)\end{array}$ & Ano & $\begin{array}{c}\text { Procedência } \\
\text { municípios }\end{array}$ & Referência \\
\hline 116 & 86,2 & 2001 a 2003 & 27 & Este artigo \\
193 & 44,0 & 1969 & 11 & Santos cols \\
222 & 60,8 & 1985 e 1986 & 19 & Dias cols $^{4}$ \\
85 & 62,7 & 1979 a 1984 & 19 & Sessa col ${ }^{11}$ \\
57 & 5,3 & 1969 & 4 & Barros cols \\
\hline
\end{tabular}

Os índices de infecção natural altos em geral apresentados por T. vitticeps podem ser atribuídos principalmente à sua relação estreita com mamíferos silvestres não refratários a $T$. cruzi ${ }^{8}$, indicando o potencial da espécie na manutenção do ciclo silvestre da doença de Chagas em sua área de ocorrência. Provavelmente a baixa incidência da doença de Chagas humana autóctone no estado seja decorrente do hábito que tem esta espécie em eliminar seus dejetos tardiamente após o repasto sanguíne $0^{39}$, visto que trabalhos sobre especificidade alimentar demonstraram presença marcante de $T$. vitticeps no intradomicílio e contato freqüente com o homem ${ }^{45}$.

Os índices de infecção natural elevados reforçam a necessidade de se manter a vigilância entomológica em áreas de ocorrência deste vetor, e estudos sobre a ecologia desta espécie devem ser conduzidos de modo que seja melhor conhecido seu habitat e fonte alimentar, além do ciclo silvestre da doença.

\section{AGRADECIMENTOS}

Ao Professor Aloísio Falqueto pela revisão e leitura crítica do manuscrito e comentários essenciais.

\section{REFERÊNCIAS BIBLIOGRÁFICAS}

1. Barros GC, Mayrink W, Abreu Salgado AA, Barros RCG, Sessa PA. Contribuição para o conhecimento da doença de Chagas autóctone no estado do Espírito Santo. Revista do Instituto de Medicina Tropical de São Paulo 17: 319-329, 1975. 
2. Camargo ME, Silva GR, Castilho EA, Silveira AC. Inquérito sorológico de prevalência da infecção chagásica no Brasil, 1975-1980. Revista do Instituto de Medicina Tropical de São Paulo 26: 179-236, 1984.

3. Dias E. Observações sobre eliminação de dejeções e tempo de sucção em alguns triatomíneos sul-americanos. Memórias do Instituto Oswaldo Cruz 54: 115-124, 1956.

4. Dias JCP, Feitosa VR, Filho ANF, Rodrigues VLC, Alencar AS, Sessa PA. Fonte alimentar e potencial vetorial de Triatoma vitticeps (Stal, 1859) com relação à doença de Chagas humana no estado do Espírito Santo, Brasil. Memórias do Instituto Oswaldo Cruz 84: 165-173, 1989.

5. Goncalves TCM, Rocha DS, Cunha RA. Feeding patterns of Triatoma vitticeps in the State of Rio de Janeiro, Brazil. Revista de Saúde Pública 34: 348-352, 2000.

6. Lent H, Wygodzinsky P. Revision of the Triatominae (Hemiptera, Reduviidae) and their significance as vectors of Chagas' disease. Bulletin American Museum of Natural History 163: 127-516, 1979.

7. Lorosa ES, Valente MVMP, Cunha V, Lent H, Jurberg J. Foco de doença de Chagas em Arcádia, Estado do Rio de Janeiro, Brasil. Memórias do Instituto Oswaldo Cruz 98: 885-887, 2003.

8. Santos CB, Ferreira AL, Leite GR, Ferreira GEM, Rodrigues AAF, Falqueto A. Peridomiciliary colonies of Triatoma vitticeps (Stal, 1859) (Hemiptera,
Reduviidae, Triatominae) infected with Trypanosoma cruzi in rural areas of the State of Espírito Santo, Brazil. Memórias do Instituto Oswaldo Cruz 100: 471-473, 2005.

9. Santos CB, Leite GR, Sessa PA, Ferreira AL, Ferreira GEM, Falqueto A. Potencial Vetorial de Triatoma vitticeps Stal, 1859 (Hemiptera, Reduviidae, Triatominae) na transmissão do Trypanosoma cruzi Chagas, 1909 (Kinetoplastidae, Trypanosomatidae) no Estado do Espírito Santo, Brasil. Revista da Sociedade Brasileira de Medicina Tropical 37: (supl I): 186, 2004.

10. Santos UM, Pinto AFS, Almeida AZ, Zanganelli FL, Carrancho PV, Netto AN Doença de Chagas no Estado do Espírito Santo - III - Vetores do tripanosoma. Revista da Sociedade Brasileira de Medicina Tropical 3: 51-52, 1969.

11. Sessa PA, Carias VRD. Infecção natural de triatomíneos do Espírito Santo for flagelados morfologicamente semelhantes ao Trypanosoma cruzi. Revista da Sociedade Brasileira de Medicina Tropical 19: 99-100, 1986.

12. Sessa PA, Pimentel RR, Ferreira AL, Falqueto A. Soroprevalência da doença de Chagas em crianças em idade escolar do Estado do Espírito Santo, Brasil, em 1999-2000. Caderno de Saúde Pública 18: 1765-1769, 2002.

13. Sherlock IA. Vetores. In: Brener Z, Andrade Z (eds) Trypanosoma cruzi e Doença de Chagas. Editora Guanabara Koogan, Rio de Janeiro, p.42-88, 1979. 